\title{
Wormholes and flux tubes in 5D Kaluza-Klein theory
}

\author{
V. Dzhunushaliev* \\ Department of Physics VCU, Richmond, Virginia 23284-2000 \\ and Theoretical Physics Department KSNU, 720024, Bishkek, Kyrgyzstan
}

D. Singleton ${ }^{\dagger}$

Department of Physics, CSU Fresno, 2345 East San Ramon Avenue M/S 37 Fresno, California 93740-8031

(Received 3 August 1998; published 12 February 1999)

\begin{abstract}
In this paper spherically symmetric solutions to 5D Kaluza-Klein theory, with "electric" and/or "magnetic" fields, are investigated. It is shown that the global structure of the spacetime depends on the relation between the "electrical" and "magnetic" Kaluza-Klein fields. For a small "magnetic" field we find a wormholelike solution. As the strength of the "magnetic" field is increased relative to the strength of the "electrical" field, the wormholelike solution evolves into a finite or infinite flux tube depending on the strength of the two fields. For the large "electric" field case we conjecture that this solution can be considered as the mouth of a wormhole, with the $G_{55}, G_{5 t}$, and $G_{5 \varphi}$ components of the metric acting as the source of the exotic matter necessary for the formation of the wormhole's mouth. For the large "magnetic" field case a 5D flux tube forms, which is similar to the flux tube between two monopoles in type-II superconductors, or the hypothesized color field flux tube between two quarks in the QCD vacuum. [S0556-2821(99)07504-9]
\end{abstract}

PACS number(s): $04.50 .+\mathrm{h}$

\section{INTRODUCTION}

Spherically symmetric metrics in multidimensional (MD) gravity can describe black holes and wormholes (WHs) (see, for example, [1-5]). Usually these papers investigate metrics without off-diagonal components. However, these components of the MD metric can play an important role as a result of the following theorem $[6,7]$.

Let $G$ be the group fiber of the principal bundle. Then there is a one-to-one correspondence between the $G$-invariant metrics on the total space $\mathcal{X}$ and the triples $\left(g_{\mu \nu}, A_{\mu}^{a}, h \gamma_{a b}\right)$. Here $g_{\mu \nu}$ is the Einstein's pseudo Riemannian metric on the base, $A_{\mu}^{a}$ is the gauge field of the group $G$ (the nondiagonal components of the multidimensional metric), and $h \gamma_{a b}$ is the symmetric metric on the fiber.

This theorem suggests that including the off-diagonal components of the MD metric is equivalent to including gauge fields [U(1), SU(2), or SU(3) gauge fields] and a scalar field $\phi\left(x^{\mu}\right)$ which is connected with the linear size of the extra dimension. These geometrical fields can act as the source of the exotic matter necessary for the formation of the wormhole's mouth. Such solutions were obtained in Refs. [8-11]. These solutions are spherically symmetric WH-like metrics with a finite longitudinal size. The throat of these WH-like solutions is located between two surfaces where the reduction from $5 \mathrm{D}$ to $4 \mathrm{D}$ spacetime breaks down. These results indicate that the exotic matter necessary for the formation of the WH can appear in vacuum multidimensional gravity from the off-diagonal elements of the metric (the gauge fields) and from the $G_{55}$ component of the metric (the scalar field), rather than coming from some externally given exotic

\footnotetext{
*Email address: dzhun@freenet.bishkek.su

${ }^{\dagger}$ Email address: das3y@maxwell.phys.csufresno.edu
}

matter. One possible application of this 5D wormhole is to "sew" two Reissner-Nordström solutions on to the two surfaces of the 5D WH solution where dimensional reduction from 5D to 4D breaks down. In this manner one obtains two asymptotically flat 4D regions with electric flux, which are connected by a 5D WH throat [12]. The splitting off or compactification of the extra dimensions is taken to occur at the surfaces where the two 4D Reissner-Nordström solutions are connected to the 5D WH throat. This composite, asymptotically flat WH has regions with both compactified extra dimensions (the two exterior regions of the 4D ReissnerNordström solutions) and with noncompactified extra dimensions (the 5D throat or bridge which connects the two $4 \mathrm{D}$ solutions). The $5 \mathrm{D}$ region of this composite $\mathrm{WH}$ has a strong gravitational field.

In Refs. [10,11] a MD metric with only "electric" fields was investigated. In Ref. [13] a MD metric with "magnetic" field $=$ "electrical" field was investigated. In this paper we investigate the consequence of having both "electric" and "magnetic" Kaluza-Klein fields of varying relative strengths. We will consider 5D Kaluza-Klein theory as gravity on the principal bundle with U(1) fiber and $4 \mathrm{D}$ space as the base of this bundle [11].

\section{INITIAL EQUATIONS}

For our spherically symmetric 5D metric we take

$$
\begin{aligned}
d s^{2}= & e^{2 \nu(r)} d t^{2}-r_{0}^{2} e^{2 \psi(r)-2 \nu(r)} \\
& \times[d \chi+\omega(r) d t+n \cos \theta d \varphi]^{2} \\
& -d r^{2}-a(r)\left(d \theta^{2}+\sin ^{2} \theta d \varphi^{2}\right),
\end{aligned}
$$

where $\chi$ is the $5^{\text {th }}$ extra coordinate, $r, \theta, \varphi$ are $3 \mathrm{D}$ sphericalpolar coordinates, $n$ is an integer, and $r \in\left\{-R_{0},+R_{0}\right\}\left(R_{0}\right.$ may be equal to $\infty)$. We require that all functions 
$\nu(r), \psi(r)$, and $a(r)$ should be even functions of $r$ and hence $\nu^{\prime}(0)=\psi^{\prime}(0)=a^{\prime}(0)=0$. According to the abovementioned theorem $\omega(r)$ is the $t$ component of the electromagnetic potential and $(n \cos \theta)$ is the $\varphi$ component. This means that we have radial Kaluza-Klein "electrical" and "magnetic", fields.

Substituting this ansatz into the 5D Einstein vacuum equations

$$
R_{A B}-\frac{1}{2} G_{A B} R=0
$$

(where $A, B=0,1,2,3,4$ ) gives us (using a REDUCE package for symbolic calculations)

$$
\begin{aligned}
& \nu^{\prime \prime}+\nu^{\prime} \psi^{\prime}+\frac{a^{\prime} \nu^{\prime}}{a}-\frac{1}{2} r_{0}^{2} \omega^{\prime 2} e^{2 \psi-4 \nu}=0, \\
& \omega^{\prime \prime}-4 \nu^{\prime} \omega^{\prime}+3 \omega^{\prime} \psi^{\prime}+\frac{a^{\prime} \omega^{\prime}}{a}=0, \\
& \frac{a^{\prime \prime}}{a}+\frac{a^{\prime} \psi^{\prime}}{a}-\frac{2}{a}+\frac{Q^{2}}{a^{2}} e^{2 \psi-2 \nu}=0, \\
& \psi^{\prime \prime}+\psi^{\prime 2}+\frac{a^{\prime} \psi^{\prime}}{a}-\frac{Q^{2}}{2 a^{2}} e^{2 \psi-2 \nu}=0, \\
& \nu^{\prime 2}-\nu^{\prime} \psi^{\prime}-\frac{a^{\prime} \psi^{\prime}}{a}+\frac{1}{a}-\frac{a^{\prime 2}}{4 a^{2}}-\frac{1}{4} r_{0}^{2} \omega^{\prime 2} e^{2 \psi-4 \nu} \\
& -\frac{Q^{2}}{4 a^{2}} e^{2 \psi-2 \nu}=0
\end{aligned}
$$

here the Kaluza-Klein 'magnetic', charge is $Q=n r_{0}$. The Kaluza-Klein "electrical', field can be defined by multiplying Eq. (4) by $4 \pi r_{0}$ and rewriting it in the following way:

$$
\left(r_{0} \omega^{\prime} e^{3 \psi-4 \nu} 4 \pi a\right)^{\prime}=0 .
$$

This can be compared with the normal 4D Gauss law

$$
\left(E_{4 D} S\right)^{\prime}=0,
$$

where $E_{4 D}$ is a $4 \mathrm{D}$ electrical field and $S=4 \pi r^{2}$ is the area of that two-sphere $S^{2}$. These are five equations for determining the four ansatz functions $(\nu, \psi, a, \omega)$. The first four equations [Eqs. (3)-(6)] are dynamical equations which determine the ansatz functions, while the last equation [Eq. (7)] contains no new dynamical information not contained in the first four equations, but gives some initial conditions related to solving this system of equations. For the metric given in Eq. (1), $r^{2}$ is replaced by $a(r)$ and the surface area is given by $S$ $=4 \pi a(r)$. Comparing Eq. (8) with Eq. (9) we can identify the 5D Kaluza-Klein "electric" field as

$$
E_{K K}=r_{0} \omega^{\prime} e^{3 \psi-4 \nu}
$$

If we integrate Eq. (8) once and let the integration constant be $4 \pi q$, then from Eq. (10) we find that $E_{K K}=q / a(r)$ where $q$ can be taken as the Kaluza-Klein "electric" charge. Finally for the system of equations given in Eqs. (3)-(7) we will consider solutions with the boundary conditions $a(0)$ $=1, \psi(0)=\nu(0)=0$ [for numerical calculations we will introduce dimensionless function $a(r) \rightarrow a(r) / a(0)$ and $x$ $=r / a(0)]$. Using these boundary conditions in Eq. (7) and also in Eq. (10) [which gives $r_{0} w^{\prime}(0)=q$ ] gives the following relationship between the Kaluza-Klein "electric'" and "'magnetic"' charges:

$$
1=\frac{q^{2}+Q^{2}}{4 a(0)} .
$$

From Eq. (11) it is seen that the charges can be parametrized as $q=2 \sqrt{a(0)} \sin \alpha$ and $Q=2 \sqrt{a(0)} \cos \alpha$.

We will examine the following different cases: (A) $Q$ $=0$ or $H_{K K}=0$, "magnetic" field is zero; (B) $q=0$ or $E_{K K}$ $=0$, "electrical" field is zero; (C) $H_{K K}=E_{K K}$, "electrical" field equal to "magnetic", field; (D) $H_{K K}<E_{K K}$, "magnetic" field less than "electrical;", (E) $H_{K K}>E_{K K}$, "electrical"' field less than "magnetic.,"

\section{A. Switched off "magnetic" field}

In this case we have the following solution $[8,10]$ :

$$
\begin{aligned}
a & =r_{0}^{2}+r^{2}, \\
e^{2 \nu} & =\frac{2 r_{0}}{q} \frac{r_{0}^{2}+r^{2}}{r_{0}^{2}-r^{2}}, \\
\psi & =0, \\
\omega & =\frac{4 r_{0}}{q} \frac{r}{r_{0}^{2}-r^{2}} .
\end{aligned}
$$

This WH-like spacetime has an asymptotical flat metric, bounded by two surfaces at $r= \pm r_{0}$ where the reduction from 5D to 4D spacetime breaks down. As $r$ moves away from 0 the cross-sectional size of the throat, $a(r)$, increases.

A connection can be made between the present solution and Wheeler's old proposal of electric charge as a wormhole filled with electric flux that flows from one mouth to the other-the "charge without charge" model of electric charge. In a recent work [12] a model of electric charge along these lines was proposed where electric charge is modeled as a kind of composite WH with a quantum mechanical splitting off of the $5^{\text {th }}$ dimension. The 5D WH-like solution of Eqs. (12)-(15) has two Reissner-Nordström black holes attached to it on the surfaces at $\pm r_{0}$. By considering 4D electrogravity as a 5D Kaluza-Klein theory in the initial Kaluza formulation with $G_{55}=1$ we can join the 5D and Reissner-Nordström solutions at the $r= \pm r_{0}$ surfaces base to base and fiber to fiber. 


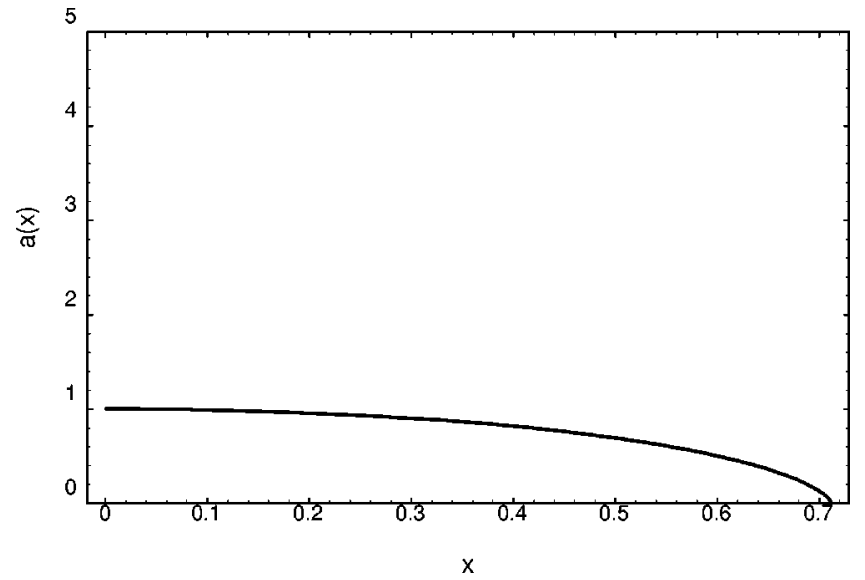

FIG. 1. Plot of the numerically evaluated ansatz function $a(x)$ for zero "electric" field. The singularities, which are taken to indicate the locations of the $\pm Q$ "magnetic" charges, occur near +0.71 and by reflection near -0.71 . To get the full picture of the flux tube one should reflect this figure about the $x$ and $y$ axes.

\section{B. Switched off "electrical" field}

In this case we will simplify by taking $\nu=0$ in addition to $\omega=0$ so that the equations reduce to

$$
\begin{array}{r}
\frac{y^{\prime \prime}}{y}+\frac{y^{\prime} a^{\prime}}{y a}-\frac{Q^{2} y^{2}}{2 a^{2}}=0, \\
\frac{a^{\prime \prime}}{a}+\frac{y^{\prime} a^{\prime}}{y a}-\frac{2}{a}+\frac{Q^{2} y^{2}}{a^{2}}=0, \\
\frac{a^{\prime} y^{\prime}}{a y}-\frac{1}{a}+\frac{a^{\prime 2}}{4 a^{2}}+\frac{Q^{2} y^{2}}{4 a^{2}}=0,
\end{array}
$$

where $y(r)=\exp [\psi(r)]$. These are three equations for two ansatz functions $\psi(r), a(r)$. The last equation, Eq. (18), simply repeats information that is already contained in the first two equations. We solved the system of equations (16),(17) numerically, using the MATHEMATICA package, with the following initial conditions: $a(0)=a_{0}=1, a^{\prime}(0)=0, y(0)$ $=1$, and $y^{\prime}(0)=0$ (this follows from the fact that we can introduce the dimensionless variable $x=r / a_{0}$ and change $a$ $\left.\rightarrow a / a_{0}\right)$. These conditions and $\alpha=0$ fix the dimensionless "magnetic" charge as $Q=2$. The results of the numerical calculations for $a(r)$ and $y(r)$ are shown in Figs. 1 and 2. We see that there is a singularity at two points $x= \pm x_{0}$. Near these singularities we find that the ansatz functions have the following asymptotic behavior:

$$
\begin{aligned}
& y(r) \approx \frac{y_{\infty}}{\left(r_{0}-r\right)^{1 / 3}}, \\
& a(r) \approx a_{\infty}\left(r_{0}-r\right)^{2 / 3}, \\
& \frac{Q y_{\infty}}{a_{\infty}}=\frac{2}{3} .
\end{aligned}
$$

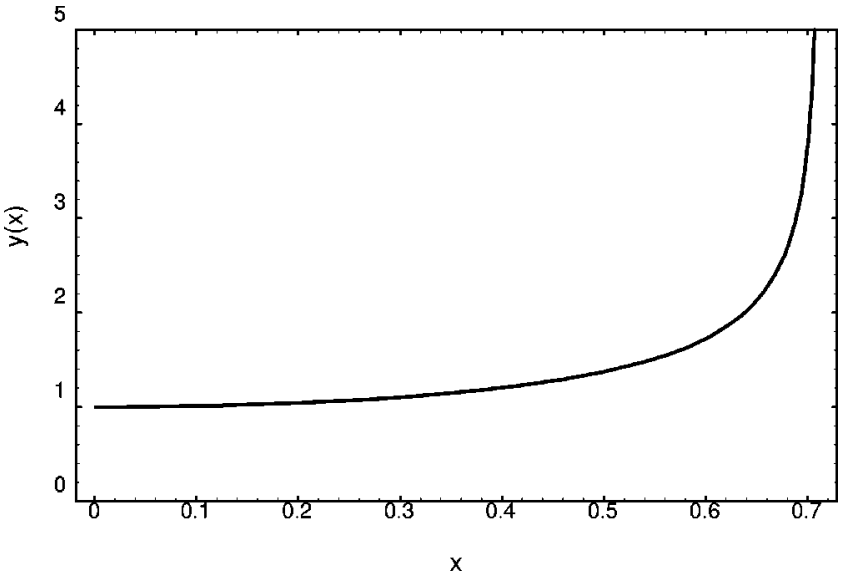

FIG. 2. Plot of the numerically evaluated ansatz function $y(x)$ for zero "electric" field.

It is interesting to note that the time part of the metric appears not to be influenced by the strong gravitational field since $G_{t t}(r)=\exp [2 \nu(r)]=1$. This result is similiar to what was found in Refs. $[14,15]$ where "magnetic" Kaluza-Klein components of the metric were considered. One difference between the present solutions and the monopole solutions of Refs. $[14,15]$ is that the monopole solutions had only coordinate singularities, while $r= \pm r_{0}$ are real singularities for the present solution. This can be seen by calculating the invariant $R_{A B} R^{A B}$ and using the asymptotic form for $y(r), a(r)$ given in Eqs. (19)-(21). This was done using a REDUCE symbolic calculation package with the result

$$
R_{A B} R^{A B} \propto \frac{1}{\left(r_{0}-r\right)^{2}} .
$$

The cross-sectional view of the ansatz function $a(r)$ of this solution is seen in Fig. 1. From this figure we take the singularities at $r= \pm r_{0}$ as the location of two magnetic charges $( \pm Q)$ with opposite signs and with flux lines of Kaluza-Klein "magnetic" field going from $+Q$ to $-Q$. It can be shown that this spacetime has a finite volume $V$, by calculating $V=\int \sqrt{-G} d^{5} v$. Near the singularities $r= \pm r_{0}$ we have

$$
\begin{aligned}
\sqrt{-G} & =\sqrt{-\operatorname{det}\left(G_{A B}\right)} \\
& =r_{0} a(r) \exp [\psi(r)] \sin \theta \approx\left(r_{0}-r\right)^{1 / 3} \rightarrow 0 .
\end{aligned}
$$

Figure 1 is very suggestive the color field flux tubes which are conjectured to form between two quarks in some pictures of confinement (see, for example, p. 548 of Ref. [16]).

In the preceding section the purely "electric" solution of Eqs. (12)-(15) can lead to a Wheeler-like model of "charge without charge"' [12]. Based on the duality between electric and magnetic charges [17] one might naively expect that a similiar Wheeler-type model for "magnetic charge without magnetic charge" should exist. However, from the $5 \mathrm{D}$ Kaluza-Klein magnetic solution of Eqs. (16)-(18) we find that the cross section of the solution given by $a(r)$ in Fig. 1 decreases as $r \rightarrow \pm r_{0}$, in contrast to the "electric'" solution 
where $a(r)$ increases as $r \rightarrow \pm r_{0}$. Furthermore, if ReissnerNordström solutions are attached to the "electric" solution as in Ref. [12], then one has a model of electric charge where the charges live in an infinite spacetime. In contrast the "magnetic" solutions are confined to a finite spacetime with a flux tube a Kaluza-Klein "magnetic" field running between the charges. This may provide a reason why free monopoles do not appear to exist in nature: they are confined into monopole-antimonopole pairs in a finite, flux-tube-like spacetime that is similiar to the flux tube confinement picture of quarks in QCD.

\section{C. "Magnetic" field equal to "electrical" field}

In this case $Q=q$ and an exact solution can be given [13]:

$$
\begin{aligned}
a & =\frac{q^{2}}{2}=\mathrm{const}, \\
e^{\psi} & =e^{\nu}=\cosh \frac{r \sqrt{2}}{q}, \\
\omega & =\frac{\sqrt{2}}{r_{0}} \sinh \frac{r \sqrt{2}}{q} .
\end{aligned}
$$

Using this solution and Eq. (10) we find that the KaluzaKlein "electrical" field is

$$
E_{K K}=\frac{q}{a}=\frac{2}{q}=\text { const. }
$$

A similiar magnetic flux-tube-like solution was discussed in Ref. [18]. The Kaluza-Klein "magnetic" field can be derived as in Refs. $[14,15]$. The gauge field associated with the metric in Eq. (1) has a $\varphi$ component as $A_{\varphi}=r_{0} n \cos \theta$. The Kaluza-Klein "magnetic" field is then found from $\mathbf{H}_{K K}$ $=\boldsymbol{\nabla} \times \mathbf{A}$, where the curl is taken using the metric of Eq. (1) and the solution of Eqs. (24)-(26). The resultant KaluzaKlein "magnetic" field derived from this has a magnitude of

$$
H_{K K}=\frac{r_{0} n}{a}=\frac{Q}{a}=\text { const. }
$$

Thus, this solution is an infinite flux tube with constant Kaluza-Klein "electrical" and "magnetic" fields. The direction of both the "electric" and "magnetic" fields is along the $\hat{\mathbf{r}}$ direction (i.e., along the axis of the flux tube). The sources of these Kaluza-Klein fields (5D "electrical" and "magnetic" charges) are located at $\pm \infty$. This feature leads us to consider this solution as a kind of 5D "electrical" and "magnetic" dipole.

\section{Intermediate cases}

We consider two different cases $E_{K K}>H_{K K}$ (or $q>Q$ ) and $E_{K K}<H_{K K}($ or $q<Q)$. The initial conditions for both cases are taken as $\psi(0)=\nu(0)=0, \psi^{\prime}(0)=\nu^{\prime}(0)=0$ and $a(0)=1, a^{\prime}(0)=0$. These initial conditions along with a choice of $\alpha$ determine the charges $q, Q$. The task of numeri-

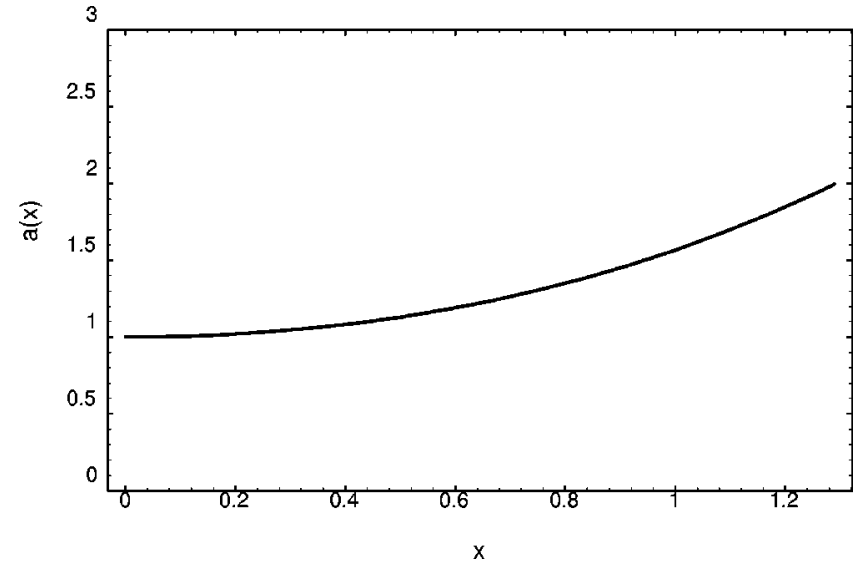

FIG. 3. Plot of the numerically evaluated ansatz function $a(r)$ for $\alpha=\pi / 3$ (i.e., the $E_{K K}>H_{K K}$ case). This solution represents a 5D WH-like throat. The "electric" and "magnetic" charges are taken to be located at the surfaces near \pm 1.24 . To get the full picture of the WH one should reflect this figure about the $x$ and $y$ axes.

cally solving the system of four equations (3)-(6) for the ansatz functions can be simplified by noting that Eq. (4) can be integrated out as we did in Sec. II. Using $E_{K K}=q / a(r)$ and Eq. (10) we find

$$
\omega^{\prime}=\frac{q}{r_{0} a(r)} e^{4 \nu-3 \psi} .
$$

In this way the $\omega$ equation has been integrated away and we can replace the $\omega^{\prime}$ term in Eq. (3) using Eq. (29), thus reducing the original system of four equations to three.

$$
\text { 1. } E_{K K}>H_{K K}
$$

The result of a numerical calculation for $a(r)$, using the MATHEMATICA package, is presented in Fig. 3 where we have taken $\alpha=\pi / 3$ so that $q>Q$. The function $e^{\nu(r)}$ is similiar in form to the function $y(r)$ in Fig. 2, and it has singularites near $\pm r_{0}= \pm 1.24$. As the "magnetic" field increases from 0 to $H_{K K}=E_{K K}$ we find the following: First, compared to the WH-like solution of the pure "electric" case, the longitudinal distance between the surfaces at $\pm r_{0}$ is stretched as the magnetic field strength increases; second, the cross-sectional size of the solution, represented by the function $a(r)$, does not increase as rapidly as $r \rightarrow \pm r_{0}$. In the limit where the "magnetic" field equals the "electrical" field, $H_{K K}=E_{K K}$, the longitudinal length of the solution goes to $\infty$ and the cross-sectional size becomes a constant.

\section{2. $\boldsymbol{E}_{K K}<\boldsymbol{H}_{K K}$}

The result of a numerical calculation is presented in Fig. 4 where we have taken $\alpha=\pi / 6$ so that $q<Q$. In this case the "electrical" field is taken as decreasing from the $E_{K K}$ $=H_{K K}$ case down to $E_{K K}=0$. As the "magnetic" field strength increases relative to the "electric" field strength we notice the following evolution of the solution: the infinite flux tube of the equal field case turns into a finite flux tube when $E_{K K}$ drops below $H_{K K}$. Also the cross-sectional size of 


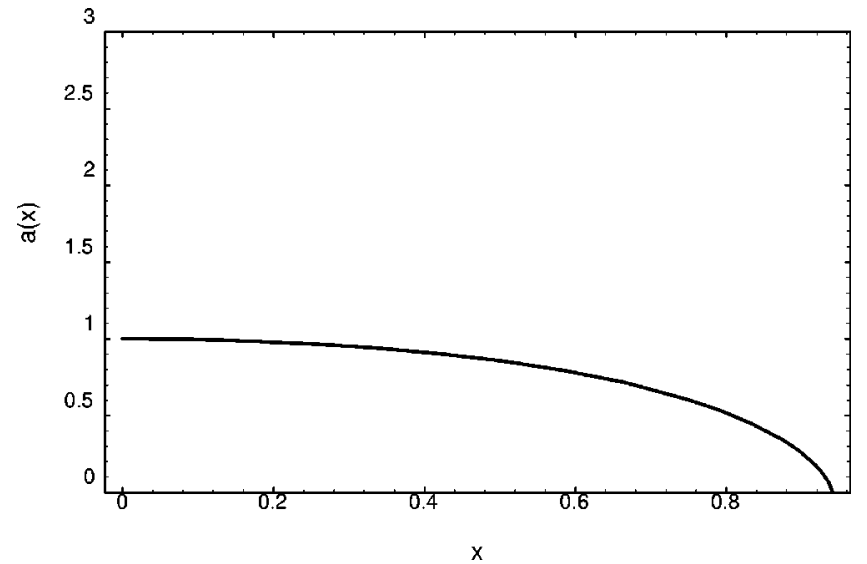

FIG. 4. Plot of the numerically evaluated ansatz function $a(r)$ for $\alpha=\pi / 6$ (i.e., the $H_{K K}>E_{K K}$ case). This figure (after reflection about the $x$ and $y$ axes) represents a flux tube with the "electric" and "magnetic" charges located at \pm 0.87 . Note that relative to the $E_{K K}=0$ case the singularities have moved further apart. This was a general feature of the solutions (i.e., the distance between the singularities increased as the strength of $E_{K K}$ increased).

this case has a maximum at $r=0$ and decreases as $r \rightarrow \pm r_{0}$ where singularities occur. We take these singularities as the locations of the "electric"/"magnetic" charges. Between the charges there is a flux tube of Kaluza-Klein "electric" and "magnetic" fields. The longitudinal size of this flux tube (the distance between charges) reaches its minimum in the limit when there is only a "magnetic', field $\left(E_{K K}=0\right)$

\section{DISCUSSION}

As the relative strengths of the Kaluza-Klein fields are varied we find that the solutions to the metric in Eq. (1) evolve in a very interesting and suggestive way. Starting with the case when there is no "magnetic" field this evolution can be sketched as follows.

(1) $H_{K K}=0$. The solution is a WH-like object located between two surfaces at $\pm r_{0}$ where the reduction of $5 \mathrm{D}$

to $4 \mathrm{D}$ spacetime breaks down. The cross-sectional size of this solution increases as $r$ goes from 0 to $\pm r_{0}$. The throat between the $\pm r_{0}$ surfaces is filled with "electric" flux.

(2) $0<H_{K K}<E_{K K}$. The solution is again a WH-like object. The throat between the surfaces at $\pm r_{0}$ is filled with both "electric" and "magnetic" fields. The longitudinal distance between the $\pm r_{0}$ surfaces increases, and the crosssectional size does not increase as rapidly as $r \rightarrow r_{0}$, compared to the previous case.

(3) $H_{K K}=E_{K K}$. In this case the solution is an infinite flux tube filled with constant "electrical" and "magnetic'" fields, and with the charges disposed at $\pm \infty$. The cross-sectional size of this solution is constant ( $a=$ const). Essentially, as the magnetic field strength is increased one can think that the two previous solutions are stretched so that the $\pm r_{0}$ surfaces are taken to $\pm \infty$ and the cross section becomes constant.

(4) $0<E_{K K}<H_{K K}$. In this case we have a finite flux tube

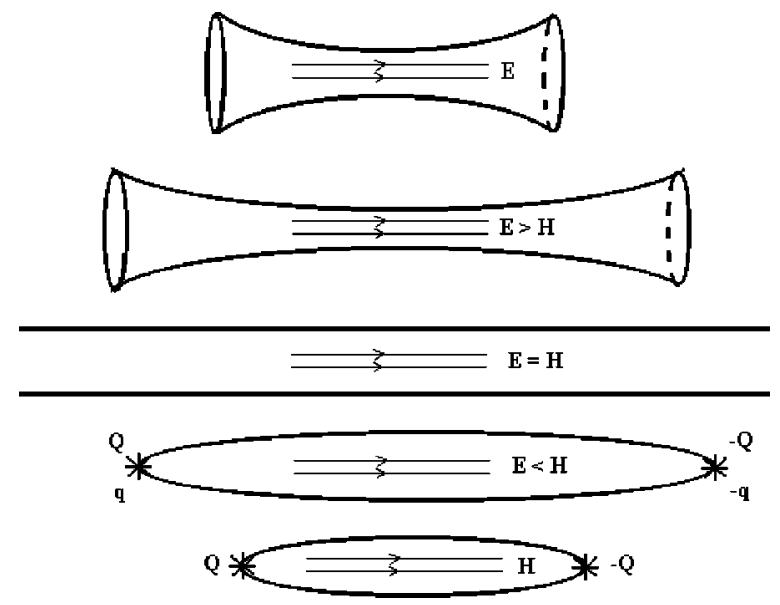

FIG. 5. The evolution from WH-like solution to finite flux tube solution. Starting from the top where $H_{K K}=0$ and the solution is WH-like, we find that as the strength of the "magnetic" field is increased the solution evolves into a stretched WH-like configuration, an infinite flux tube, a finite flux tube, and finally for $E_{K K}$ $=0$ into a finite flux tube with minimal separation between the charges.

located between two $(+)$ and $(-)$ "electrical" and "magnetic" charges located at $\pm r_{0}$. Thus the longitudinal size of this object is again finite, but now the cross sectional size decreases as $r \rightarrow r_{0}$. At $r= \pm r_{0}$ this solution has real singularities which we interpret as the locations of the charges. This solution is very similar to the confinement mechanism in QCD where two quarks are disposed at the ends of a flux tube with color electrical and magnetic fields running between the quarks. In this connection one can ask if this similarity is accidental or if there is some deeper connection between 5D Kaluza-Klein gravity and QCD. We note that in Ref. [19] some mappings between 4D gravity and nonAbelian theory are discussed.

(5) $E_{K K}=0$. This solution is again a finite flux tube only with a "magnetic" field filling the flux tube. In this solution the two opposite "magnetic" charges are confined to a spacetime of fixed volume. This may indicate why single, asymptotic magnetic charges have never been observed in nature: they are permanently confined to monopoleantimonopole pairs of some fixed volume.

The evolution of the solution from a WH-like object, to an infinite flux tube, to a finite flux tube, as the relative strengths of the fields is varied, is presented in Fig. 5. This allows us to make two complimentary conclusions: First, if one takes some Wheeler-like model of electric charge as in Ref. [12], then it can be seen that if the magnetic field becomes too strong, the WH-like solution is destroyed and with it the Wheeler-like model of electric charge. Second, if one concentrates a sufficiently strong electric field (i.e., $E_{K K}$ $>H_{K K}$ ) into some small region of spacetime, one is led to the science-fiction-like possibility that one may be able to "open' the finite flux tubes into a WH-like configuration. This conjecture assumes some kind of spacetime foam model where the vacuum is populated by virtual flux tubes filled with virtual "magnetic", and/or "electric" fields. 
Starting from the solutions obtained here we see that in 5D gravity there is a distinction between "electrical" and "magnetic"' Kaluza-Klein fields. This can be contrasted with the 4D electrogravity Reissner-Nordström solution which is the same for the electrical and magnetic charges.

\section{ACKNOWLEDGMENTS}

This work has been funded by the National Research Council under the Collaboration in Basic Science and Engineering Program.
[1] M. S. Morris and K. S. Thorne, Am. J. Phys. 56, 395 (1988).

[2] S. Giddings and A. Strominger, Nucl. Phys. B306, 890 (1988).

[3] K. A. Bronnikov and J. C. Fabris, Grav. Cosmol. 3, 67 (1997).

[4] M. Rainer and A. Zhuk, Phys. Rev. D 54, 6186 (1996).

[5] M. Visser, Lorentzian Wormholes: From Einstein to Hawking (AIP, New York, 1995).

[6] A. Salam and J. Strathdee, Ann. Phys. (N.Y.) 141, 316 (1982).

[7] R. Percacci, J. Math. Phys. 24, 807 (1983).

[8] A. Chodos and S. Detweiler, Gen. Relativ. Gravit. 14, 879 (1982).

[9] G. Clément, Gen. Relativ. Gravit. 16, 131 (1984).

[10] V. D. Dzhunushaliev, Izv. Vuzov, Fiz. 6, 78 (1993); Grav. Cosmol. 3, 240 (1997).
[11] V. D. Dzhunushaliev, Gen. Relativ. Gravit. 30, 583 (1998).

[12] V. D. Dzhunushaliev, Mod. Phys. Lett. A 13, 2179 (1998).

[13] V. D. Dzhunushaliev and D. Singleton, "Flux Tube Solution in 5D Kaluza-Klein Theory,'” gr-qc/9805104.

[14] D. J. Gross and M. J. Perry, Nucl. Phys. B226, 29 (1983).

[15] R. Sorkin, Phys. Rev. Lett. 51, 87 (1983).

[16] Michael E. Peskin and Daniel V. Schroeder, An Introduction to Quantum Field Theory (Addison-Wesley, New York, 1995).

[17] J.D. Jackson, Electrodynamics (Wiley, New York, 1975), p. 252.

[18] A. Davidson and E. Gedalin, Phys. Lett. B 339, 304 (1994).

[19] D. Singleton, Phys. Lett. A 223, 12 (1996). 